\title{
Comparison for Image Edge Detection Algorithms
}

\author{
Li Bin, Mehdi Samiei yeganeh \\ ${ }_{1,2}$ (School of Information Technology, Jawaharlal Nehru Technological University, China, Iran)
}

\begin{abstract}
Edge is the basic characteristic of image, edge detection plays an important role in computer vision and image analysis. The pretty usefull and identical information contained in edge of sub-image enable edge detection to be the main approach to image analysis and recognition. This paper compares and analyzes several kinds of classical algorithms of image edge detection, including Roberts, Sobel, Prewitt, LOG and Canny with MATLAB tool.
\end{abstract}

Keywords - Canny, LOG, Prewitt, Roberts, Sobel

\section{INTRODUCTION}

There are two goals for image processing: one is to obtain the image that more suitable for human observing and understanding, the other one is to recognize the image automatically by computer. The key step is to decompose a large and complex image into small image with independent feature.

The edge is the basic characteristic of image. It is a collection of pixels whose surrounding pixels have a grayscale step-like changes or changes in the roof. The edge widely exists between objects and background, objects and primitives. It contains rich information, step property, shape etc, which is able to describe the target object. There are two types of edge detection: one is step change edge whose pixels grayscale of two sides have significantly difference; the other one is roof edge that is the turning point from increase to decrease of gray value.

Edge is basically the symbol and reflection of discreteness of partial image ${ }^{[1]}$. It symbolizes the end of one area and the beginning of the other area. The detected edge may become wide or discrete with the existence of noisy and ambiguity. So what we have to do for edge obtaining is to detect the discreteness of partial image and then eliminate breaking points of edges. Complete edge is combined by these edge pixels.

\section{PRINCIPLE OF EDGE DETECTION}

Edge detection operator is a mutation in the nature of the image edge to test the edge. There are two main types $^{[2]}$ : one is the first derivative-based edge detection operator to detect image edges by computing the image gradient values, such as Roberts operator, Sobel operator, Prewitt operator; the other one is the second derivative-based edge detection operator, by seeking in the second derivative zero-crossing to edge detection, such as LOG operator, Canny operator.

\subsection{Based ON The First ORder Derivative Of Edge Detection}

Gradient is a measure of the function changes. And it is also the first order derivative of the image corresponds to two-dimensional function. An image can be seen as a continuous derivative of image intensity of sampling points group. Gradient ${ }^{[3]}$ is a type of two-dimensional equivalent of the first derivative. So it can be defined as a vector.

$$
\mathrm{G}(\mathrm{x}, \mathrm{y})=\left[\begin{array}{l}
\mathrm{G}_{\mathrm{x}} \\
\mathrm{G}_{\mathrm{y}}
\end{array}\right]=\left[\begin{array}{l}
\partial \mathrm{f} / \partial \mathrm{x} \\
\partial \mathrm{f} / \partial \mathrm{y}
\end{array}\right]
$$

There are two important properties. First, the vector $\mathrm{G}(\mathrm{x}, \mathrm{y})$ direction is same as the direction of the maximum rate of change of increasing function $\mathrm{f}(\mathrm{x}, \mathrm{y})$ ( eg. formula(2) ); Second, the gradient amplitude ( eg.formula (3));

$$
\begin{aligned}
& |\mathrm{G}(\mathrm{x}, \mathrm{y})|=\sqrt{G_{x}{ }^{2}+G_{y}{ }^{2}} \\
& \propto(\mathrm{x}, \mathrm{y})=\arctan \left(\mathrm{G}_{\mathrm{x}} / \mathrm{G}_{\mathrm{y}}\right)
\end{aligned}
$$

For digital images, partial derivative of the edge is almost same as differences. So the edge often lies on the differential value of the maximum, minimum, or zero.

$G_{x}=f[x+1, y]-f[x, y]$

$G_{y}=f[x, y+1]-f[x, y]$

When we calculate the gradient, the same location $(x, y)$ of real partial derivatives is essential in computing space. Gradient approximation is not in the same location using the above formula. So the $2 \times 2$ firstorder differential template is used to calculate partial derivatives in $\mathrm{x}$ and $\mathrm{y}$ direction of the interpolation points $[x+1 / 2, y+1 / 2]$, then Gx and Gy can be expressed as:

$\mathrm{G}_{\mathrm{x}}=\left[\begin{array}{ll}-1 & 1 \\ -1 & 1\end{array}\right]$ 
$\mathrm{G}_{\mathrm{y}}=\left[\begin{array}{cc}1 & 1 \\ -1 & -1\end{array}\right]$

\subsection{Based On The First Order Derivative Of Edge Detection}

The first order derivative method described above uses a boundary point. This method may lead to the edge points to detect excessive data storage. Theoretically more effective way is to determine the point with maximum value in ladder and these points are to be considered as edge point.

This method can detect more precise edge. The first derivative of the local maximum corresponds to the second derivative zero-crossing point. So more accurate edge points can be found by the determination of the zero crossing point of the second derivative of the image gray.

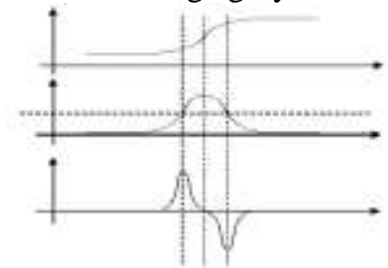

Fig. 1: The image of the second derivatives

\subsection{Roberts operator}

\section{Algorithms OF EdGE DETECTION}

Roberts $^{[4]}$ operator is a first-order operator, which uses a partial differential operator to find the edge. It uses the approximation between the two adjacent pixels of the diagonal direction of the gradient amplitude to detect edge. In the field of $2 x 2$ diagonal derivative, the two convolution kernels, respectively; $G_{x}=\left[\begin{array}{cc}1 & 0 \\ 0 & -1\end{array}\right], G_{y}$ $=\left[\begin{array}{cc}0 & 1 \\ -1 & 0\end{array}\right]$ as shown in Figure 2. Roberts operator is defined as:

$G(x, y)=\left\{[\sqrt{f(x, y)}-\sqrt{f(x+1, y+1)}]^{2}+[\sqrt{f(x+1, y)}-\sqrt{f(x, y+1)}]^{2}\right\}^{1 / 2}$

Gradient size of Roberts operator represents the edge strength of the edge and direction of the gradient and the edge are vertical. The operator edge has higher positioning accuracy, but it is easy to lose a part of the edge. The operator with a steep low-noise image corresponds best.

\begin{tabular}{|l|l|}
\hline 1 & 0 \\
\hline 0 & -1 \\
\hline 0 & 1 \\
\hline-1 & 0 \\
\hline
\end{tabular}

Fig. 2: Roberts Operator

\subsection{Sobel operator}

Sobel operator ${ }^{[5]}$ is in the form of the filtering operator. It is used to extract the edge. Each point in the image are the two nuclear convolutions. One checks maximum response of the vertical edge, and the other one checks maximum response of the horizontal edge. The maximum valueof two convolutions will be referred as output value of the changing point. Sobel operator is easy to achieve in space, has a smoothing effect on the noise, is nearly affected by noise, can provide more accurate edge direction information but it will also detect many false edges with coarse edge width.

\begin{tabular}{|l|l|l|}
\hline-1 & -2 & -1 \\
\hline 0 & 0 & 0 \\
\hline 1 & 2 & 1 \\
\hline
\end{tabular}

\begin{tabular}{|l|l|l|}
\hline-1 & 0 & 1 \\
\hline-2 & 0 & 2 \\
\hline-1 & 0 & 1 \\
\hline
\end{tabular}

\subsection{Prewitt operator}

Fig. 3: Sobel Operator

The Prewitt operator is one type of an edge model operator. Fig. 5 shows that two convolution kernels formed Prewitt operator. Model operator is made from the ideal edge sub-image composition. Detect the image using edge model one by one, and take the maximum value of the model operator that is most similar to the detected region as the output of the operator. Both Prewitt operator and Sobel operator use the same differential and filtering operations, the only difference is that the template does not use the same image.

\subsection{LOG operator}


LOG (Laplacian of Gaussian) operator find the optimal filter of edge detection by ratio of the signal to noise of image. Firstly, a Gaussian function is used to low-pass smoothingly filter image; then high-pass filter the Laplacian operator, according to the second derivative of zero to detect the edges. Gaussian filter function ${ }^{[8]}$ is:

$$
\mathrm{G}(\mathrm{x}, \mathrm{y}, \sigma)=\frac{1}{2 \pi \sigma^{2}} \exp \left(-\frac{1}{2 \sigma^{2}}\left(\mathrm{x}^{2}+\mathrm{y}^{2}\right)\right)
$$

Where letter is the standard deviation of the Gaussian filter, which determines the degree of smoothing of the image. By The low-pass filtering the image $\mathrm{f}(\mathrm{x}, \mathrm{y})$ we can get $\mathrm{f}(\mathrm{x}, \mathrm{y}) * \mathrm{G}(\mathrm{x}, \mathrm{y}, \sigma)$ and $\mathrm{g}(\mathrm{x}, \mathrm{y})=\nabla^{2}[\mathrm{f}(\mathrm{x}, \mathrm{y}) * \mathrm{G}(\mathrm{x}, \mathrm{y}, \sigma)]=\mathrm{f}(\mathrm{x}, \mathrm{y}) * \nabla^{2} \mathrm{G}(\mathrm{x}, \mathrm{y}, \sigma)$

Where $\nabla^{2} \mathrm{G}$ is LOG operator.

\subsection{Canny operator}

Canny proposed three criteria of the evaluation the pros and cons of performance of edge detection: (1) standard of ratio of signal to noise, that is real edge detection probability is higher and non-edge points sentenced to be lower the probability of edge points, so that the output of ratio of signal to noise is maximum; (2) standard of positioning accuracy, that is there is great possibility that the detected edge points is actually in center of the edge; (3) The unilateral corresponding standard, that is the probability of multiple response in single edge is low, and false edge The response should be the maximum inhibition.

Canny operator ${ }^{[6]}$ is based on three criteria. The basic idea uses a Gaussian function to smooth image firstly. Then the maximum value of first derivative also corresponds to the minimum of the first derivative. In other words, both points with dramatic change of gray-scale (strong edge) and points with slight change of grayscale (weak edges) correspond to the second derivative zero-crossing point. Thus these two thresholds are used to detect strong edges and weak edges. The fact that Canny algorithm is not susceptible to noise interference enables its ability to detect true weak edges.

\section{COMPARISON OF EDGE DETECTION}

This paper use MATLAB ${ }^{[7]}$ to evaluate these algorithms by setting different thresholds.

4.1 The result of Roberts operator:

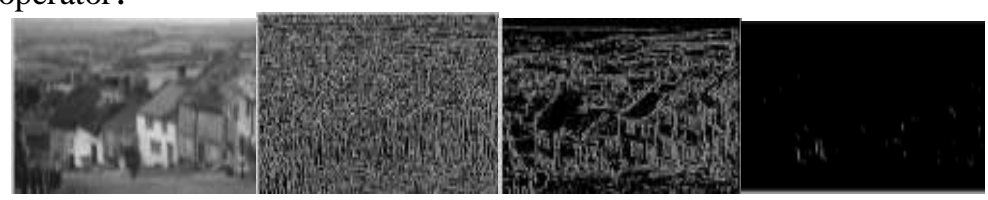

(a)

(b)

(c)

(d)

Fig. 4 :.( a) original image (b) threshold $=0.00$ (c) threshold $=0.05$ (d) threshold $=0.3$

4.2 The result of Sobel operator:

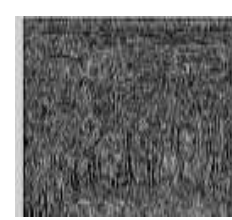

(a)

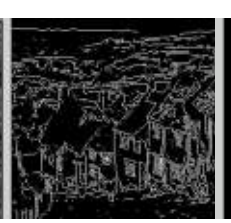

(b)

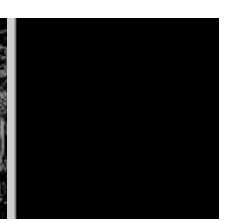

(c)

Fig. 5: (a) threshold $=0.00$ (b) threshold $=0.05$ (c) threshold $=0.3$

4.3 The result of Prewitt operator:

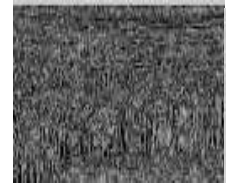

(a)

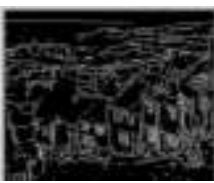

(b)

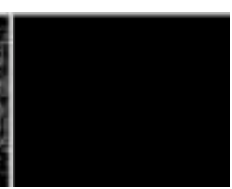

(c)

Fig. 6: (a) threshold $=0.00$ (b) threshold $=0.05$ (c) threshold $=0.3$

4.4 The result of Canny operator:

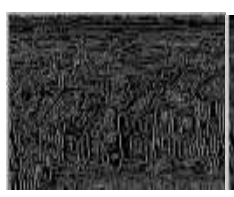

(a)

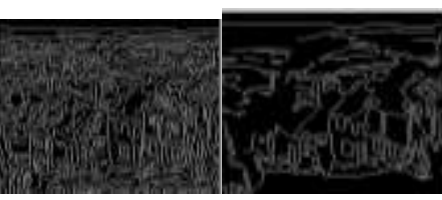

(c)

Fig. 7: (a) threshold $=0.00$ (b) threshold $=0.05$ (c) threshold $=0.3$ 
Result analysis: figure 4, 5, 6,7 are the result of first order derivative of edge detection, The greater the threshold is, the clearer image edge processing effect is and the more coherent the edge points are significant. However, when the threshold is over 0.3, the effective information of the image edge will be lost. We can see that Canny algorithm is best among all algorithms. Because Canny can filter noise and maintain the integrity of valid information. Canny operator also can ensure high positioning accuracy of the image ${ }^{[8]}$. And other operators are more sensitive to noise than Canny, and cannot be filtered.

4.5 The result of LOG operator:

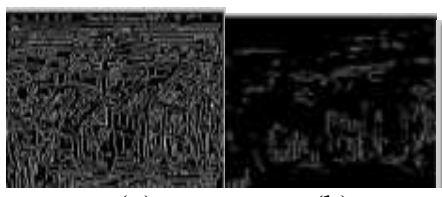

(a)

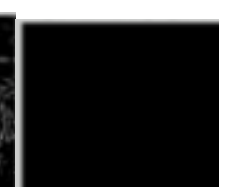

(c)

Fig. 8: (a) threshold $=0.00$ (b) threshold $=0.01$ (c) threshold $=0.3$

Result analysis: Fig. 8 is the result of second order derivative of edge detection, the smaller the threshold is, the clearer marginal treatment effect of the image is, more coherent the edge points are significant. LOG algorithm is sensitive to noise by using the algorithm of image intensity second order derivative zero crossing point. Therefore, it should do denoising before enhance the image.

It's very necessary to detect pseudo-edge caused by noise because detection accuracy can be improved; In order to improve noise immunity, position deviation takes place. Actual image contains noise, and noise distribution, variance, and other information that are unknown to us. Smoothing filter operation to eliminate noise with high-frequency signal, but detected edge shifts.

Due to factor of physical and light the edge of the actual image often has different scales, and each edge pixel scale is unknown. It's not possible to detect edge very well by using a single fixed-scale edge detection operator.

Classical edge detection methods ${ }^{[10]}$ are extremely sensitive to noise due to the introduction of various forms of differential operation. Noise is detected as edge points in edge detection instead of real edge with interference of noise. Thus a good edge detection method should have good noise immunity and outstanding property of restraining noises which are the advantages of Canny operator.

\section{CONCLUSION}

One-dimensional operator Roberts, Sobel and Prewitt are able to handle treatment effect of images of more gray-scale gradient and noise. The Sobel operator is more sensitive to the diagonal edge is than to the horizontal and vertical edges. On the contrary, Prewitt operator is more sensitive to horizontal and vertical edges.

LOG often produces the edge of double pixels wide; therefore, LOG operator is rarely directly used for edge detection. It is mainly used to determine pixels to determine if the pixels of image are in the dark areas or bright area of the known edge.

Canny operator is based on three criteria. The basic idea uses a Gaussian function to smooth image firstly. Then the maximum value of first derivative also corresponds to the minimum of the first derivative. In other words, both points with dramatic change of gray-scale (strong edge) and points with slight change of grayscale correspond to the second derivative zero-crossing point. Thus these two thresholds are used to detect strong edges and weak edges. The fact that Canny algorithm is not susceptible to noise interference enables its ability to detect true weak edges. Canny algorithm is not susceptible to noise interference enables its ability to detect true weak edges. It's optimal edge detection algorithm.

\section{Journal Papers:}

\section{REFERENCES}

[1] L.P. Han and W.B. Yin. An Effective Adaptive Filter Scale Adjustment Edge Detection Method(China, Tsinghua university, 1997).

[2] D. Marr and E. Hildreth, Theory of Edge Detection(London, 1980).

[3] Q.H Zhang, S Gao, and T.D Bui, Edge detection models, Lecture Notes in Computer Science, 32(4), 2005, $133-140$.

[4] D.H Lim, Robust Edge Detection In Noisy Images, Computational Statistics \& Data Analysis, 96(3), $2006,803-812$.

[5] Abbasi TA, Abbasi MU, A novel FPGA-based architecture for Sobel edge detection operator, International Journal of Electronics, 13(9), 2007, 889-896.

[6] Canny John, A Computational Approach to Edge Detection, IEEE Transactions on Pattern Analysis and Machine Intelligence, PAMI-8(6), 1986, 679-6987

[7] X.L Xu, Application of Matlab In Digital Image Processing, Modern Computer, 43(5), 2008, 35-37.

[8] Y.Q Lv and G.Y Zeng, Detection Algorithm of Picture Edge, TAIYUANSCIENCE \& TECHNOLOGY, 27(2), 2009, 34-35

[9] D.F Zhang, MATLAB Digital Image Processing(Beijing, Mechanical Industry, 2009) 